\title{
Comunicação
}

[Communication]

\section{Ocorrência de Actinobacillus pleuropneumoniae biótipo 1 em pulmões de suínos com pleuropneumonia no Norte de Portugal}

\author{
[Occurrence of Actinobacillus pleuropneumoniae biotype 1 in swine with pleuropneumonia \\ in the North of Portugal] \\ F.J. Vieira-Brito ${ }^{1}$, M.G. Vieira-Brito ${ }^{1}$, A.C. Coelho ${ }^{1,2^{*}}$, J. Rodrigues ${ }^{1,2}$ \\ ${ }^{1}$ Departamento de Ciências Veterinárias \\ Universidade de Trás-os-Montes e Alto Douro \\ 5001-911 - Vila Real, Portugal \\ ${ }^{2}$ CECAV Portugal
}

A pleuropneumonia, causada por Actinobacillus pleuropneumoniae, é uma das mais importantes doenças respiratórias suínas que ocorre em quase todos os países, acarretando elevadas perdas econômicas em todas as idades (Losinger, 2005). A enfermidade está normalmente associada com fatores ambientais e de manejo (Perestrelo-Vieira et al., 2000). São conhecidos dois biótipos: o biótipo 1 é do tipo NAD dependente, tendo sido descritos 15 sorotipos. A classificação dos resultados das lesões pulmonares durante a inspecção é usada para caracterizar o problema de doença calculando a ocorrência de tipos específicos de doença pulmonar, como pleuropneumonia (Andreasen et al., 2001). Embora esta seja, normalmente, causada por $A$. pleuropneumoniae, outras bactérias do gênero, como Haemophilus, assim como bactérias "Pasteurella-like", estão associadas em alguns casos (Nicolet, 1992). Um diagnóstico correto de A. pleuropneumoniae necessita de exames patológicos e bacteriológicos post-mortem para a identificação da etiologia da pleuropneumonia. A demonstração de $A$. pleuropneumoniae viável por cultura bacteriológica ainda é a técnica mais usada em muitos laboratórios (Gottschalk et al., 2003). As provas fundamentadas na genética apresentam-se muito interessantes, contudo, não parecem ser práticas nem econômicas para o processamento de grande número de amostras (Turni e Blackall, 2007).

Para este estudo, foram colhidas 127 amostras de pulmão em matadouro, provenientes de cinco explorações intensivas em ciclo fechado no Norte de Portugal. De todos os pulmões efetuouse uma valorização macroscópica externa minuciosa sendo tomada nota da presença, ausência e características das lesões quando presentes. Do total de amostras, 96 apresentaram alterações anatomopatológicas compatíveis com pleuropneumonia e 31 amostras não tinham qualquer alteração macroscópica visível.

As amostras foram classificadas em dois grupos de acordo com a presença ou ausência de lesões típicas compatíveis com pleuropneumonia. O critério de inclusão em cada grupo baseou-se no método de classificação semiquantitativo utilizado pelo ITP Institut Technique du Porc (ITP) descrito por Le Foll e Solignac (1988) (Tab. 1). De acordo com esta classificação, cada lobo pulmonar é classificado com uma nota que varia entre zero e quatro. Fez-se ainda a classificação das lesões de pleurisia de acordo com a mesma grelha de apreciação de lesões.

Recebido em 31 de março de 2008

Aceito em 20 de outubro de 2008

*Autor para correspondência (corresponding author)

E-mail: accoelho@utad.pt 


\section{Ocorrência de Actinobacillus...}

Tabela 1. Classificação semiquantitativa das lesões pulmonares (pneumonia e pleurisia)

\begin{tabular}{|c|c|c|}
\hline Nota & Pneumonia & Pleurisia \\
\hline 0 & Ausência de lesão & Ausência de lesão \\
\hline 1 & $\begin{array}{l}\text { Lesões cobrindo menos de } 1 / 4 \text { da } \\
\text { superfície do lobo }\end{array}$ & Pequeno foco de pleurisia visceral \\
\hline 2 & $\begin{array}{c}\text { Lesões cobrindo de } 1 / 4 \text { a metade da } \\
\text { superfície do lobo }\end{array}$ & Pleurisia visceral ou parietal limitada \\
\hline 3 & $\begin{array}{l}\text { Lesões cobrindo desde metade até } 3 / 4 \text { da } \\
\text { superfície do lobo }\end{array}$ & Lesão mais grave com algumas aderências \\
\hline 4 & $\begin{array}{l}\text { Lesões cobrindo mais de } 3 / 4 \text { da superfície } \\
\text { do lobo }\end{array}$ & Pulmão totalmente aderente \\
\hline
\end{tabular}

Fonte: adaptado de Le Foll e Solignac, 1988)

As amostras foram colhidas de forma asséptica e conservadas em refrigeração a $4^{\circ} \mathrm{C}$. Em lesões de pleuropneumonia, o fragmento é localizado na charneira entre a zona lesada e a zona sã, de acordo com o procedimento de Gutiérrez et al. (1992). Os órgãos, transportados para o laboratório, foram semeados em no máximo seis horas após a colheita. Com base na técnica aplicada por Harley e Prescott (2002), executou-se a sementeira diretamente a partir do tecido pulmonar com auxílio de uma zaragatoa. $\mathrm{O}$ isolamento primário do agente foi feito em ágar chocolate suplementado com fator $\mathrm{V}$ no qual se encontravam agentes inibidores, como a bacitracina $(100 \mu \mathrm{g} / \mathrm{ml})$ e a cloxacilina $(1 \mu \mathrm{g} / \mathrm{ml})$, com antimicrobianos (violeta de cristal $-1 \mu \mathrm{g} / \mathrm{ml}$ ). A incubação foi realizada durante 24 a 48 horas a $37^{\circ} \mathrm{C}$, em atmosfera com $10 \%$ de $\mathrm{CO}_{2}$ (Oxoid BR38). A coloração de Gram foi efetuada para a confirmação do crescimento de bacilos/cocobacilos Gram-negativos. Foi utilizada a estirpe de referência \#4074 MITTAL. As subculturas destinadas à realização de provas de identificação complementares foram efetuadas em meio PPLO enriquecido com $10 \%$ de extrato fresco de levedura e $20 \%$ de soro inativado de cavalo e $1 \%$ de ágar. Para a confirmação de A. Pleuropneumoniae, realizaram-se as provas bioquímicas de identificação: dependência de factores de crescimento; crescimento no meio de Triple Sugar Iron (TSI); reação de Christie-Atkins-MunchPeterson (CAMP); prova da catalase; prova da oxidase; pesquisa da urease; produção de indol; fermentação dos hidratos de carbono; prova da ortonitrofenol galactosídeo (ONPG); teste de lysine decarboxylase sulphydrase (LDS); teste Ornithine decarboxylase arginine dihydrolase (ODC/ADH). Os microrganismos identificados bioquimicamente foram mantidos em meio de conservação com leite desnatado.

A ocorrência observada de lesões pulmonares foi de $75,6 \%$. A ocorrência de isolamento do agente foi de 19,7\%. Em 25 (26,0\%) das 96 amostras com sinais evidentes de pleuropneumonia, isolou-se $A$. pleuropneumoniae. Quanto à localização anatômica dos diferentes tipos de lesões, observou-se que a maioria se situou no lobo cardíaco (47,9\%), seguido do lobo apical $(40,6 \%)$ e, por último, o diafragmático (20,8\%). Encontraram-se lesões de pleurisia em seis $(6,3 \%)$ pulmões lesionados. Os resultados obtidos de acordo com a classificação proposta por Le Foll e Solignac (1988) encontramse na Tab. 2.

Isolou-se A. pleuropneumoniae biótipo 1, em 25 $(19,7 \%)$. Este valor representa isolamento do agente em $26,0 \%$ dos 96 pulmões que apresentavam lesões de pleuropneumonia. Não foi isolado $A$. pleuropneumoniae das 31 amostras de pulmões que não continham lesões típicas à inspecção macroscópica.

Tabela. 2. Ocorrência das lesões nos pulmões de acordo com a classificação de Le Foll e Solignac, 1988

\begin{tabular}{lcccc}
\hline Classificação & Apical & Lobo & Pleurisia \\
\hline 0 & $57(59,4 \%)$ & $50(52,1 \%)$ & $76(79,2 \%)$ & $90(93,8 \%)$ \\
1 & $13(13,5 \%)$ & $15(15,6 \%)$ & $9(9,3 \%)$ & $4(4,2 \%)$ \\
2 & $11(11,4 \%)$ & $17(17,7 \%)$ & $4(4,2 \%)$ & $1(1,0 \%)$ \\
3 & $6(6,3 \%)$ & $7(7,3 \%)$ & $2(2,1 \%)$ & $0(0,0 \%)$ \\
4 & $9(9,4 \%)$ & $7(7,3 \%)$ & $5(5,2 \%)$ & $1(1,0 \%)$ \\
\hline
\end{tabular}


A infecção causada por $A$. pleuropneumoniae origina a identidade clínica designada por pleuropneumonia, normalmente restrita à cavidade torácica. As lesões pulmonares são freqüentemente bilaterais e englobam o lobo apical, o cardíaco e o diafragmático. Para Pointon et al. (1992), as lesões mais severas localizam-se na parte caudal dos lobos cardíaco e diafragmático. Os resultados deste estudo estão em conformidade com esses autores, na medida em que se encontrou maior percentagem de lesões no lobo cardíaco. Existe a opinião mais ou menos generalizada de que a persistência de $A$. pleuropneumoniae em uma exploração traduz-se por elevada ocorrência de pleurisias e lesões pulmonares observadas quando do abate. Neste trabalho, observou-se uma relação entre a ocorrência dessas lesões e o sucesso de isolamento de A. Pleuropneumoniae. Não se isolou este microrganismo em pulmões com ausência de lesões. Os resultados obtidos apontam para uma associação entre a presença de lesões compatíveis com pleuropneumonia e o isolamento do agente etiológico. Algumas lesões podem ter sido causadas por $A$. Pleuropneumoniae, mas não foi possível a confirmação devido à dificuldade de isolar o agente a partir de lesões crônicas (Williams et al., 2000). A percentagem de isolamento do agente nos pulmões que apresentavam lesões de pleuropneumonia foi menor que a apresentada em outros trabalhos em diferentes países (Hunter e Livingstone, 1986; Williams et al., 2000). Os resultados deste trabalho confirmam os apresentados por outros autores, sobre a validade do diagnóstico de pleuropneumonia mediante isolamento do agente em pulmões com lesões (Williams et al., 2000). No México, esses autores, isolaram A. pleuropneumoniae em 129 $(51,6 \%)$ amostras de pulmão com pleuropneumonia crônica. Os melhores resultados apresentados por esses autores podem ser atribuídos à seleção do lobo diafragmático, na medida em que, relativamente aos outros parâmetros, no presente estudo cumpriu-se toda a metodologia das técnicas, como a assepsia, e garantiu-se a qualidade dos meios de cultura para o isolamento (Brandreth e Smith, 1985). Também é possível que a ocorrência de pleuropneumonia por A . pleuropneumoniae seja mais alta no México que em Portugal e, como tal, a porcentagem de isolamento do agente também seja maior. Os resultados deste estudo reforçam a importância do isolamento e a identificação do agente em presença de lesões típicas no diagnóstico da doença.

Palavras-chave: Actinobacillus pleuropneumoniae, pleuropneumonia, isolamento, ocorrência

\begin{abstract}
The isolation and identification of Actinobacillus pleuropneumoniae in swine lungs with pleuropneumonia in the North of Portugal were reported. A total of 127 swine lungs with and without lesions were examined. The system of lesions classification was based on a semi-quantitative method. Diagnosis was made by isolation and identification of the etiological agent in typical lesions. The occurrence of observed lesions was $75.6 \%$ and the occurrence of isolation of A. pleuropneumoniae was $19.7 \%$. In 25 out of 96 (26.0\%) lung samples with lesions of pleuropneumonia, A. pleuropneumoniae was isolated.
\end{abstract}

Keywords: Actinobacillus pleuropneumoniae, pleuropneumonia, isolation, occurrence

\section{REFERÊNCIAS BIBLIOGRÁFICAS}

ANDREASEN, M.; MOUSING, J.; KROGSGAARD, L. No simple association between time elapsed from seroconversion until slaughter and the extent of lung lesions in Danish swine. Prev. Vet. Med., v.52, p.147-161, 2001.

BRANDRETH, S.; SMITH, I. Prevalence of pig herds affected by pleuropneumonia associated with Haemophilus pleuropneumoniae in eastern England. Vet Rec., v.117, p.143-147, 1985.

GOTTSCHALK, M.; BROES, A.; MITTAL, K.R. et al. Non-pathogenic Actinobacillus isolates antigenically and biochemically similar to Actinobacillus pleuropneumoniae: a novel species? Vet. Microbiol., v.92, p.87-101, 2003.

GUTTIÉRREZ, C.B.; RODRIGUEZBARBOSA, J.I.; GONZALEZ, O.R. et al. 


\section{Ocorrência de Actinobacillus...}

Viability of Actinobacillus pleuropneumoniae in frozen pig lung samples and comparison of different methods of direct diagnosis in fresh samples. Comp. Immun. Microbiol. Infect. Dis., v.15, p.89-95, 1992.

HARLEY, J.P.; PRESCOTT, L. Laboratory exercises in microbiology. 5.ed. New York: McGraw-Hill, 2002. 466p.

HUNTER, D.; LIVINGSTONE, J. Detection of Haemophilus pleuropneumoniae antigens using the coagglutination test. Vet. Rec., v.118, p.129, 1986.

LE FOLL, P.; SOLIGNAC, T. Systèmes de notation des lésions respiratoires du porc charcutier. Révue biliographique et application à l'épidémio-surveillance. Techni-Porc, v.11, p.2539, 1988.

LOSINGER, W.C. Economic impacts of reduced pork production associated with the diagnosis of Actinobacillus pleuropneumoniae on grower/finisher swine operations in the United States. Prev. Vet. Med., v.68, p.181-193, 2005.
NICOLET, J. Actinobacillus pleuropneumoniae. In: STRAW, B.E. (Ed). Diseases of swine, 7.ed. Iowa: Iowa University, 1992. p.401-408.

PERESTRELO-VIEIRA, R.; SOBESTIANSKY, J.; BARCELLOS, D. et al. Doenças dos suínos. 2.ed. Lisboa: Ciência e Vida, 2000. p.589-600.

POINTON, A.M.; MERCY, A.R.; BACSTROM, L. et al. Disease surveillance at slaughter: In: LEMAN, A.D.; STRAW, W.L.; MENGELING, W.L. et al. (Eds). Diseases of swine. 7.ed. Iowa: Iowa University, 1992. p.968-985.

TURNI, C.; BLACKALL, P.J. An evaluation of the apxIVA based PCR-REA method for differentiation of Actinobacillus pleuropneumoniae. Vet. Microbiol., v.121, p.163-169, 2007.

WILLIAMS, J.J.; TORRES-LEON, M.A.; ECHEVERRIA-COELLO, P. et al. Aislamiento e identificación de Actinobacillus pleuropneumoniae en pulmones de cerdos con pleuropneumonía crónica sacrificados en el rastro municipal de Mérida, Yucatán, México. Rev. Biomed., v.11, p.175-181, 2000. 